\title{
Effects of benzydamine and mouthwashes containing benzydamine on Candida albicans adhesion, biofilm formation, regrowth, and persistence
}

\author{
Andrea Ardizzoni ${ }^{1}$ [C $\cdot$ Giorgia Boaretto ${ }^{2} \cdot$ Eva Pericolini $^{1,2} \cdot$ Diego Pinetti $^{3} \cdot$ Alessandra Capezzone de Joannon $^{4}$. \\ Lucia Durando ${ }^{4} \cdot$ Lorella Ragni $^{4}$ - Elisabetta Blasi ${ }^{1,2}$
}

Received: 4 August 2021 / Accepted: 30 November 2021 / Published online: 23 January 2022

(C) The Author(s) 2021

\begin{abstract}
Objectives To assess the effects of benzydamine and mouthwashes (MoWs) containing benzydamine on different stages of Candida albicans biofilm: adhesion, formation, persistence, and regrowth (if perturbed).

Materials and methods C. albicans CA1398, carrying the bioluminescence ACT1p-gLUC59 fusion product, was employed. Fungal cells were exposed for $1^{\prime}, 5^{\prime}$, or $15^{\prime}$ to 4 different benzydamine concentrations $(0.075$ to $0.6 \%)$ to 2 mouthwashes (MoWs) containing benzydamine and to a placebo MoW (without benzydamine). Treated cells were tested for adhesion (90 $\mathrm{min}$ ) and biofilm formation (24-h assay). Next, 24- and 48-h-old biofilms were exposed to benzydamine and MoWs to assess regrowth and persistence, respectively. The effects of benzydamine, MoWs containing benzydamine, and placebo on different biofilm stages were quantified by bioluminescence assay and by the production of quorum sensing (QS) molecules. Results Benzydamine and MoWs containing benzydamine impaired $C$. albicans ability to adhere and form biofilm, counteracted $C$. albicans persistence and regrowth, and impaired a 48-h-old biofilm. Some of these effects paralleled with alterations in QS molecule secretion.

Conclusions Our results show for the first time that benzydamine and MoWs containing benzydamine impair C. albicans capacity to form biofilm and counteract biofilm persistence and regrowth.

Clinical relevance Benzydamine and MoWs containing benzydamine capacity to affect $C$. albicans biofilm provides an interesting tool to prevent and treat oral candidiasis. Likely, restraining C. albicans colonization through daily oral hygiene may counteract colonization and persistence by other critical oral pathogens, such as Streptococcus mutans, whose increased virulence has been linked to the presence of $C$. albicans biofilm.
\end{abstract}

Keywords Benzydamine $\cdot$ Mouthwashes $(\mathrm{MoWs}) \cdot$ Candida albicans $\cdot$ Bioluminescence $\cdot$ Biofilm $\cdot$ Adhesion

Andrea Ardizzoni

ardizzoni.andrea@unimore.it

1 Department of Surgical, Medical, Dental and Morphological Sciences with interest in Transplant, Oncological and Regenerative Medicine, University of Modena and Reggio Emilia, Via Campi, 287, 41125 Modena, Italy

2 Graduate School of Microbiology and Virology, University of Modena and Reggio Emilia, Modena, Italy

3 Centro Interdipartimentale Grandi Strumenti (C.I.G.S.), University of Modena and Reggio Emilia, Modena, Italy

4 Global R\&D PLCM-Angelini Pharma S.p.A., via Vecchia del Pinocchio 22, 60131 Ancona, Italy

\section{Introduction}

Candida albicans (C. albicans) often colonizes the oral cavity of healthy subjects, where it appears in its yeast form. Because of its dimorphism (an important virulence trait, which allows fungal switch between yeast and filamentous forms), Candida behaves as an opportunistic pathogen and upon transition to filamentous forms, it is capable of causing mucosal infections mainly in immunocompromised individuals $[1,2]$. Oropharyngeal infections by $C$. albicans do not affect only AIDS patients, but they are often related also to diabetes that, in turn, is associated with xerostomia (a salivary $\mathrm{pH}$ disorder which reduces the salivary flow [3]), oral cancer [4], and terminally ill conditions [5]. Formation of hyphae is linked to the expression of characteristic hyphae-associated genes, such 
as hyphal wall protein 1 (Hwp1), agglutinin-like sequence 3 (Als3), secreted aspartic proteases 4, 5, and 6 (Sap4, 5, and 6), and the hyphae-associated proteins extent of cell elongation protein 1 (Ece1) and hyphal regulated cell wall protein 1 (Hyr1) [6]. Since most of such molecules are adhesins, their interaction with cell surface receptors grants the fungus the capacity to bind efficiently to oral mucosal epithelia as well as teeth surface $[7,8]$. Such fungal adhesion, promoted also by hyphae formation, facilitates in turn biofilm establishment. In addition, the presence of hyphae elicits proinflammatory cytokine production and helps the fungus to avoid phagocytosis and/or intracellular killing, ultimately causing tissue damage [9]. Biofilm in particular shelters the fungal cells from the action of immune-mediated defenses [10], antifungal drugs, and disinfectants [11-14].

Interestingly, this enhanced resistance of the sessile form of the fungus is not simply due to a mere physical sheltering, but also to the possibility of the biofilm-embedded fungi to form a community, whose components can communicate with each other through the release and monitoring of low molecular weight hormone-like secreted molecules. Such mechanism is known as quorum sensing (QS) and the molecules responsible for the inter-microbial communication are collectively indicated as QS molecules [15]. Four main QS molecules have been described to date in the kingdom of Fungi: farnesol, tyrosol, phenylethanol, and tryptophol $[16,17]$. Their concentration within the biofilm environment has been reported to be proportional to the size of the fungal population: beyond a critical threshold, a response is triggered, leading to the coordinated expression or repression of QS-related genes [18]. QS molecules can regulate Candida morphogenesis (by stimulating yeast-to-hyphae or hyphae-to-yeast switching), can initiate fungal apoptosis, and can affect the fungal virulence [17]. Among QS molecules, farnesol and tyrosol are known to be involved in biofilm formation by modulating several virulence factors of the fungus including its dimorphic transition. In particular, farnesol induces hyphae-to-yeast transition and inhibits biofilm formation, whereas tyrosol has an opposite effect by stimulating hyphae production and therefore exerting a pivotal role in biofilm production [15].

In addition to yeast-to-hyphae switching, Candida cell surface hydrophobicity is another important feature that reportedly favors fungal adhesion to inert surfaces. This may represent another explanation for frequent oral infections in those individuals who harbor abiotic materials within the oral cavity for therapeutic purposes (such as acrylic denture base, orthodontic metal braces, and surface of dental restorations) [19-22]. For this same reason, oral candidiasis can easily develop in individuals (especially elderly people) who make use of dental prostheses, as well as in all those individuals/patients who fail to produce sufficient saliva [23].

Good oral hygiene practices, such as daily use of toothbrush, toothpaste, and mouthwash, are crucial in contributing to prevent oral infections by Candida [24]. As a matter of fact, we have recently demonstrated, by means of in vitro models, that both $C$. albicans hyphal development and biofilm formation and persistence can be affected by several mouthwashes, especially by those containing chlorhexidine digluconate, cetylpyridinium chloride, and essential oils in their formulations $[9,25]$.

Benzydamine hydrochloride is a nonsteroidal drug with anti-inflammatory effects mainly due to its capacity to inhibit TNF- $\alpha$ and IL- $1 \beta$ production and release $[26,27]$. Also, this molecule has analgesic and anesthetic properties [28-30]. Even though benzydamine cannot be considered an antibiotic, it has been demonstrated to have some antimicrobial effects [31]. Because of its properties, benzydamine has been included in the formulation of several mouthwashes, where its use as an anti-inflammatory drug was mainly intended to delay or prevent radiation-induced oral mucositis in patients undergoing radiation therapy [32-34]. More recently, mouthwashes and oral spray formulations, containing either $0.15 \%$ benzydamine hydrochloride or $0.2 \%$ chlorhexidine digluconate, were shown to have similar anti-inflammatory effectiveness against gingival inflammation caused by plaque accumulation [35, 36]. Notwithstanding the similarity in the anti-inflammatory activity, benzydamine was generally better tolerated and it lacked the side effects associated with the use of chlorhexidine, such as staining of teeth, loss of taste, and feeling of numbness [37]. Mouthwashes containing a combination of benzydamine hydrochloride and cetylpyridinium chloride were proven effective as well in reducing plaque formation, in comparison with mouthwashes containing cetylpyridinium alone, without any relevant clinical or microbiological adverse effect [38].

Little is known about the antifungal effects of benzydamine hydrochloride on C. albicans, especially on the fungus capacity to adhere to substrates and to form biofilm. For this reason, here we performed a series of in vitro studies, in order to assess if benzydamine hydrochloride (either alone or in combination with other molecules within commercial mouthwashes) could impair C. albicans adhesion to an abiotic substrate and its capacity to form a biofilm. Moreover, we evaluated the capacity of the molecule (and of the MoWs containing it) to disrupt mature biofilm and to interfere with its persistence and regrowth. We then assessed the effect of MoWs containing benzydamine in the production of $C$. albicans $\mathrm{QS}$ molecules, a key factor involved in both biofilm formation and biofilm persistence.

\section{Materials and methods}

\section{Reagents}

Benzydamine (1-benzyl-3-dimethylaminopropoxy-lH-indazole hydrochloride) was supplied by Angelini Pharma S.p.A. (Roma, Italy). The powder was resuspended in complete F12 medium (cF12). The latter consisted of Dulbecco's modified Eagle's 
medium/Ham's nutrient mixture F12 (Sigma, St. Louis, MO, USA), supplemented with $10 \%$ heat-inactivated fetal bovine serum (HiFBS, Defined Hyclone, Logan, UT, USA), $50 \mathrm{mg} /$ $\mathrm{ml}$ gentamicin (Bio Whittaker, Verviers, Belgium), $2 \mathrm{mg} / \mathrm{ml}$ Ciproxin (ICN Biomedicals, Cleveland, OH, USA), and 2 $\mathrm{mM}$ L-glutamine (EuroClone, Milan, Italy). The benzydamine solution was sterilized by filtration with $0.22-\mu \mathrm{m}$ syringe filters and diluted to the working strength concentrations used for the experiments (see below). Benzydamine was always resuspended and diluted fresh, on the day of the experiment.

Three different mouthwashes were also employed for the study. MoW 1 ("Tantum Verde $0.15 \%$ collutorio", Angelini Pharma S.p.A., Roma, Italy) containing $0.15 \%$ benzydamine hydrochloride and 96\% ethanol; MoW 2 ("Tantum Verde Bocca $22.5 \mathrm{mg} / 15 \mathrm{ml}+7.5 \mathrm{mg} / 15 \mathrm{ml}$ collutorio", Angelini Pharma S.p.A., Roma, Italy) containing $0.15 \%$ benzydamine hydrochloride, $0.05 \%$ cetylpyridinium chloride, and 96\% ethanol; MoW 3 (supplied by Angelini Pharma S.p.A., Roma, Italy) containing only ethanol and used as a placebo. MoWs 1 and 2 have been commercially available for several years; they are safe and not toxic (further information on this topic can be found in the supplementary materials section).

\section{Fungal strain}

The BLI Candida albicans strain employed in this study was the CA1398, carrying the bioluminescence ACT1pgLUC59 fusion product [39]. To assess the effects of different benzydamine concentrations and contact times as well as the effects of different MoWs employed for different contact times on $C$. albicans viability, fungi were counted to a working strength concentration of $2 \times 10^{6} / \mathrm{ml}$ and resuspended in $\mathrm{cF} 12$ medium (see above).

\section{MIC determination}

The benzydamine MIC for BLI-Candida was evaluated according to the broth microdilution method NCCLS M27 [40]. The data obtained were confirmed by reading the optical density (O.D.) at 540-nm wavelength, by means of a plate reader (Tecan Sunrise, Austria).

\section{Experimental design}

\section{Protocol for adhesion and biofilm production}

In order to evaluate the effects of benzydamine on Candida capacity to adhere to plastic and to form a biofilm, fungal cells $\left(2 \times 10^{6} / \mathrm{ml}\right.$ in $\mathrm{cF} 12$ medium) were treated for 1,5 , or 15 min with 4 different concentrations of benzydamine (6 $\mathrm{mg} / \mathrm{ml}(0.6 \%), 3 \mathrm{mg} / \mathrm{ml}(0.3 \%), 1.5 \mathrm{mg} / \mathrm{ml}(0.15 \%)$, and 0.75 $\mathrm{mg} / \mathrm{ml}(0.075 \%))$, with the $3 \mathrm{MoWs}$, or with cF12 medium alone (used as a control). After treatment, fungal cells were washed, dispensed in a black 96-well microtiter plate (PerkinElmer Life Sciences, Cambridge, UK) $(0.1 \mathrm{ml} /$ well, in triplicate), and placed at $37{ }^{\circ} \mathrm{C}$ with $5 \% \mathrm{CO}_{2}$. Incubation was carried out for $90 \mathrm{~min}$ (for adhesion assessment) or for $24 \mathrm{~h}$ (for biofilm formation assessment).

\section{Protocol for biofilm regrowth}

To establish the effects of benzydamine on biofilm regrowth, Candida cells ( $2 \times 10^{6} / \mathrm{ml}$ in cF12 medium) were dispensed in a black 96 -well microtiter plate $(0.1 \mathrm{ml} /$ well, in triplicate $)$ and placed at $37{ }^{\circ} \mathrm{C}$ with $5 \% \mathrm{CO}_{2}$ for $24 \mathrm{~h}$, to allow biofilm production. Then, Candida was exposed to the concentrations of benzydamine indicated above, to the MoWs, or to $\mathrm{cF} 12$, for 1,5 , or $15 \mathrm{~min}$. Finally, the wells were gently washed with PBS, $100 \mu 1$ of fresh $\mathrm{cF} 12$ was added to each well, and the plate was placed back at $37^{\circ} \mathrm{C}$ with $5 \% \mathrm{CO}_{2}$ and incubated for additional $24 \mathrm{~h}$, prior to be assessed for microbial regrowth.

\section{Protocol for biofilm persistence}

To evaluate the effects of benzydamine and MoWs on a mature biofilm, Candida cells $\left(2 \times 10^{6} / \mathrm{ml}\right.$ in cF12 medium) were dispensed in a black 96-well microtiter plate $(0.1 \mathrm{ml} /$ well, in triplicate) and placed at $37{ }^{\circ} \mathrm{C}$ with $5 \% \mathrm{CO}_{2}$ for 48 $\mathrm{h}$, to allow production of a mature biofilm. Then, Candida was exposed to the concentrations of benzydamine indicated above, to the MoWs, or to $\mathrm{cF} 12$ for 1,5 , or $15 \mathrm{~min}$, prior to be assessed for residual biofilm. The timeline of each experimental protocol is detailed in Fig. 1.

\section{Bioluminescence analysis}

After incubation at $37{ }^{\circ} \mathrm{C}$ with $5 \% \mathrm{CO}_{2}$ (carried out for different times, according to the parameter investigated, as detailed in the previous paragraph), the supernatants were collected and stored at $-80{ }^{\circ} \mathrm{C}$ for the HPLC-ESI/HRMS analysis (see below). Then, the wells were gently washed 3 times with PBS. Then, $2 \mu \mathrm{M}$ coelenterazine (SynChem, $\mathrm{Ohm}$, Germany) in luciferase assay buffer (LA buffer) was added and the relative luminescence units (RLU) were assessed by immediately reading the plate with a Fluoroskan FL luminometer (Thermo Scientific, Waltham, MA, USA).

\section{Fungal viability measurement}

Candida suspensions ( $0.5 \mathrm{ml} /$ tube $)$ were exposed to benzydamine $(0.5 \mathrm{ml} /$ tube; at different concentrations $)$ or to the 
A
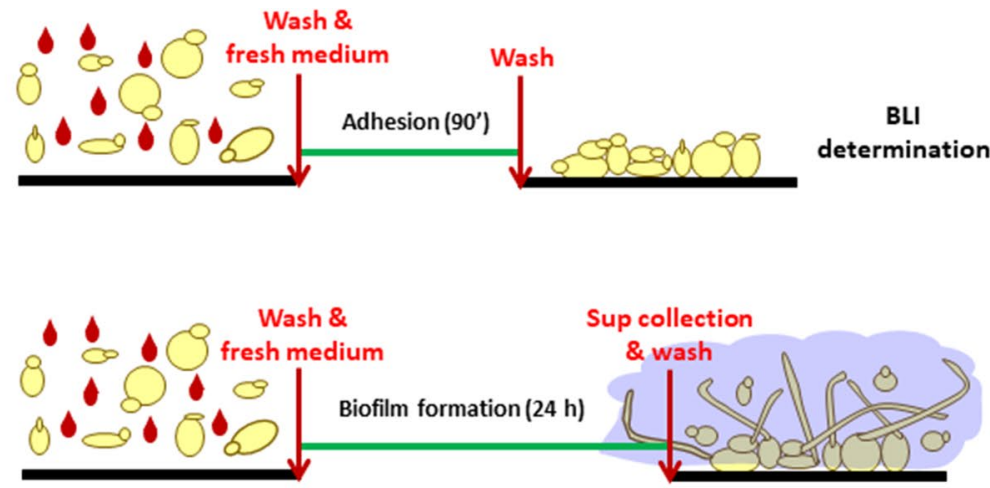

BLI determination \&

QS molecules analysis

C
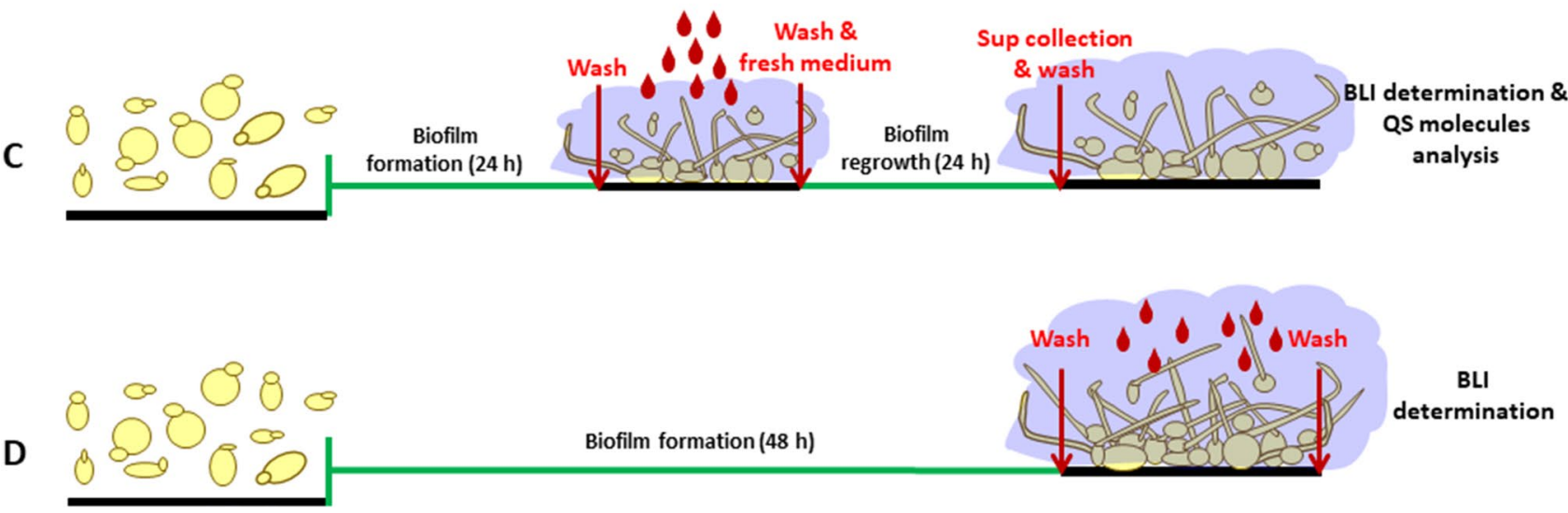

Fig. 1 Experimental design performed to assess the effects of benzydamine and MoWs on BLI-Candida adhesion (A), biofilm formation (B), 24-h-old biofilm regrowth (C), and 48-h-old biofilm persistence (D). BLI-Candida cells, incubated for 1, 5, or 15 min with different concentrations of benzydamine or different MoWs, were seeded in 96-well plates. Adhesion was assessed after 90 min of incubation (A) and the capacity to form a biofilm was evaluated after $24 \mathrm{~h}$ of incuba-

MoWs $(0.5 \mathrm{ml} /$ tube $)$; the tubes were incubated at $37^{\circ} \mathrm{C}$ with $5 \% \mathrm{CO}_{2}$ for 1,5 , or $15 \mathrm{~min}$. As controls, Candida suspensions ( $0.5 \mathrm{ml} /$ tube) were added to $0.5 \mathrm{ml}$ of $\mathrm{cF} 12$ and incubated at $37{ }^{\circ} \mathrm{C}$ with $5 \% \mathrm{CO}_{2}$ for the same times. After each time of contact, the tubes were centrifuged at $4500 \mathrm{rpm}$ for 8 min, washed with PBS, centrifuged again, and suspended with cF12. Serial dilutions of Candida suspensions were then seeded in SDA plates to evaluate the growth inhibition capacity of different benzydamine concentrations and of the different MoWs formulations per se.

\section{Assessment of the quorum sensing (QS) molecules by liquid chromatography-electrospray/ high-resolution mass spectrometry (HPLC-ESI/ HRMS)}

After MoWs containing benzydamine treatment, the detection of 2 quorum sensing (QS) molecules, farnesol and tyrosol, was performed by HPLC-ESI/HRMS. Tyrosol tion (B). To test the capacity of biofilm regrowth, a 24-h-old biofilm was treated with different concentrations of benzydamine or different MoWs and further incubated up to $48 \mathrm{~h}$ (C). Finally, the effects of different benzydamine concentrations and different MoWs on biofilm persistence were observed by treating a 48-h-old BLI-Candida biofilm (D). The results of all these experiments were achieved by bioluminescence assay

(2-(4-hydroxyphenyl)-ethanol) and farnesol (3,7,11-trimethyl-2,6,10-dodecatriene-1-ol) standard solutions were supplied by Sigma-Aldrich (St. Louis, MO, USA). Both reagents were reconstituted to $1 \mathrm{mg} / \mathrm{ml}$ in $95 \%$ methanol (Carlo Erba Reagents, Milan, Italy). External standard calibration samples were prepared in water:methanol 95:5 (v/v) to cover the 0.5 to $1000 \mathrm{ng} / \mathrm{ml}$ concentration range for both analytes.

The levels of tyrosol and farnesol were measured in supernatants collected from untreated controls and samples treated for 15 min with the MoWs, after a further incubation for $24 \mathrm{~h}$ (biofilm formation) and $48 \mathrm{~h}$ (biofilm regrowth). The supernatants, which had been stored at -80 ${ }^{\circ} \mathrm{C}$, were thawed and centrifuged at $14,000 \mathrm{rpm}$ for 10 min, in order to get rid of cellular debris. Then, they were transferred to Amicon-Ultra 0.5 tubes (Sigma-Aldrich, St. Louis, MO, USA) and centrifuged again at $14,000 \mathrm{rpm}$ for $15 \mathrm{~min}$. Prior to be analyzed, such samples were diluted $1: 1(\mathrm{v} / \mathrm{v})$ with $5 \%$ methanol in water and transferred to the autosampler pending analysis. 
Analyses were performed on an Ultimate 3000 HPLC connected to a Q Exactive high-resolution mass spectrometer via a HESI-II electrospray ionization source (Thermo Scientific), controlled by Xcalibur software (Thermo Scientific, v. 29 build 2926). A 10- $\mu$ l volume of sample solution was injected onto a Hypersil Gold C18 $100 \times 2.1 \mathrm{~mm}$ ID $1.8 \mu \mathrm{m}$ ps column (Thermo Scientific, Waltham, MA, USA) kept at $30{ }^{\circ} \mathrm{C}$ and separation was performed at $0.4 \mathrm{ml} / \mathrm{min}$ flow with a gradient elution scheme using methanol (B) and $0.1 \%$ formic acid in water (A). The mobile phase composition was kept at $2 \% \mathrm{~B}$ for 0.2 min after injection then linearly raised to $42 \% \mathrm{~B}$ in $15 \mathrm{~min}$ and further on to $98 \% \mathrm{~B}$ in $3.3 \mathrm{~min}$. Methanol was kept at $98 \%$ up to minute 24.9 then lowered to $2 \%$ at minute 25 . The total runtime was $35 \mathrm{~min}$. ESI source was operated in positive ionization mode. Capillary temperature was set at $320{ }^{\circ} \mathrm{C}$; the following nitrogen flows (arbitrary units) were used to assist the ionization: Sheath Gas 40, Aux Gas 30 (at $290^{\circ} \mathrm{C}$ ), Sweep Gas 3. The capillary voltage was set to $3.8 \mathrm{kV}$ and S-Lens RF level was set at 45 (arbitrary units).

Tyrosol and farnesol were monitored in positive ionization mode using targeted SIM (tSIM) experiments with a 3 -min window (retention time $\pm 1.5 \mathrm{~min}$ ), $0.5 \mathrm{amu}$ isolation window (target $m / z \pm 0.25 \mathrm{amu}$ ), AGC target value of 2E5, 140000 FWHM resolution (at $\mathrm{m} / \mathrm{z} 200$ ), and maximum ion injection time of $503 \mathrm{~ms}$. In-source CID at $8 \mathrm{eV}$ was used during tyrosol detection.

The molecular protonated ion $\left([\mathrm{M}+\mathrm{H}]^{+}\right)$was observed for tyrosol at $121.06479 \mathrm{~m} / \mathrm{z}$ while farnesol was detected by means of its most abundant in-source fragment at 205.19508 $\mathrm{m} / \mathrm{z}$ due to loss of water from its molecular protonated ion $\left(\left[\mathrm{M}+\mathrm{H}-\mathrm{H}_{2} \mathrm{O}\right]^{+}\right)$.

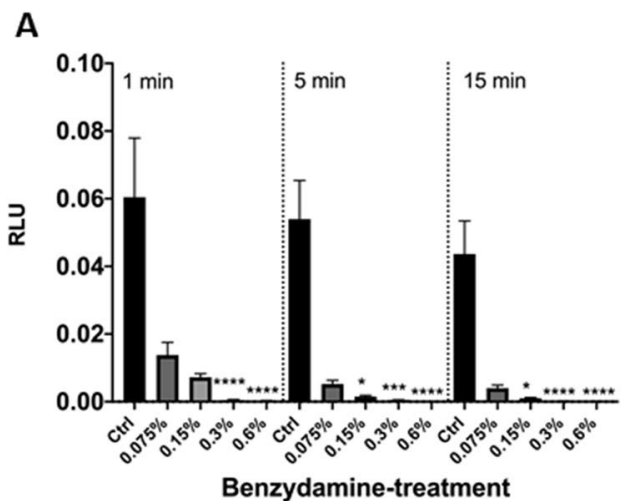

Fig. 2 Effects of benzydamine (A) and MoWs (B) treatments on BLICandida adhesion. Candida cells, pretreated or not with different concentrations of benzydamine or with different MoWs for 1, 5, or $15 \mathrm{~min}$, were assessed for the capacity to adhere to plastic, by bioluminescence assay. The results are shown as mean values \pm standard errors of triplicate samples from three different experiments. ${ }^{*} p \leq 0.05$, $* * * p<0.001$,

\section{Statistical analyses}

All data are from 3 different experiments, with triplicate samples. Quantitative variables were tested for normal distribution by the Shapiro-Wilk test. Statistical differences between groups were analyzed according to the KruskalWallis followed by Dunn's multiple comparisons test by using GraphPad Prism 8 . Values of $* p \leq 0.05,{ }^{* *} p \leq$ $0.01, * * * p \leq 0.001$, and $* * * * p \leq 0.0001$ were considered significant.

\section{Results}

\section{Effects of benzydamine and MoWs containing benzydamine on C. albicans adhesion}

By using the bioluminescence assay, the fungal capacity to adhere to plastic was assessed, using a previously described protocol [9]. Candida cells incubated for $90 \mathrm{~min}$ in medium alone (Ctrl) returned the highest bioluminescent signal, indicating the strong capacity of the fungus per se to adhere to the plastic microwells. As shown in Fig. 2A, by pre-treating Candida with the highest benzydamine concentrations, i.e., $0.6 \%$ and $0.3 \%$, the bioluminescent signals were always reduced in a highly significant fashion, irrespective of the contact times. The pretreatment of Candida with $0.15 \%$ benzydamine determined a reduction of the bioluminescent signals, which became significant only after 5- and 15-min contact times. Finally, by pre-treating Candida with the lowest benzydamine dose, i.e., $0.075 \%$, a consistent (albeit not significant) reduction of bioluminescence signal could be observed.

\section{B}

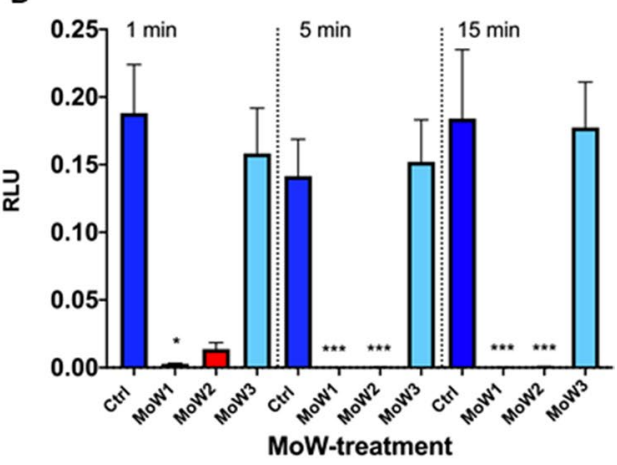

or $* * * * p<0.0001$. RLU, relative luminescence units. MoW 1 ("Tantum Verde $0.15 \%$ collutorio") contains $0.15 \%$ benzydamine hydrochloride and 96\% ethanol; MoW 2 ("Tantum Verde Bocca $22.5 \mathrm{mg} / 15 \mathrm{ml}+$ $7.5 \mathrm{mg} / 15 \mathrm{ml}$ collutorio") contains $0.15 \%$ benzydamine hydrochloride, $0.05 \%$ cetylpyridinium chloride, and $96 \%$ ethanol; MoW 3 (placebo) contains only ethanol 
In a second set of experiments, the effect of commercial MoWs containing $0.15 \%$ benzydamine (albeit with different formulations) was assessed in comparison to placebo MoW without benzydamine on Candida adhesion to plastic surface. Again, Candida cells incubated for $90 \mathrm{~min}$ in medium alone (Ctrl) always returned the highest bioluminescent signal. A trend very similar to the control Candida samples could be observed when the fungal cells were pretreated with MoW 3, used as a placebo. Differently, the pretreatment of Candida with MoWs 1 and 2 caused a highly significant reduction of the bioluminescent signals, irrespective of the contact times (Fig. 2B).

\section{Effects of benzydamine and MoWs containing benzydamine on the capacity of $C$. albicans to form a biofilm}

The bioluminescence assay was employed to assess the capacity of benzydamine- and MoWs-pretreated Candida cells to produce a biofilm. Fungal cells, incubated for $24 \mathrm{~h}$ in medium alone ( $\mathrm{Ctrl}$ ), returned the highest bioluminescent signal, indicating a strong capacity of the fungus per se to efficiently form a biofilm. By pre-treating Candida with the highest benzydamine doses, i.e., $0.6 \%$ and $0.3 \%$, the reduction in bioluminescent signals was significantly higher compared to the controls, irrespective of the contact times. Moreover, by pre-treating Candida with $0.15 \%$ benzydamine, the bioluminescent signals were reduced as well, but statistical significance was reached only after 15-min contact time (Fig. 3A).

By pre-treating Candida with MoW 1, the bioluminescent signals were partly reduced at $15 \mathrm{~min}$ with respect to the control, even though statistical significance could not be reached. Differently, the pretreatment of Candida with MoW 2 caused a significant reduction in bioluminescent signals after $15 \mathrm{~min}$ of pretreatment, with respect to both control Candida and Candida treated with placebo MoW 3 (Fig. 3B).

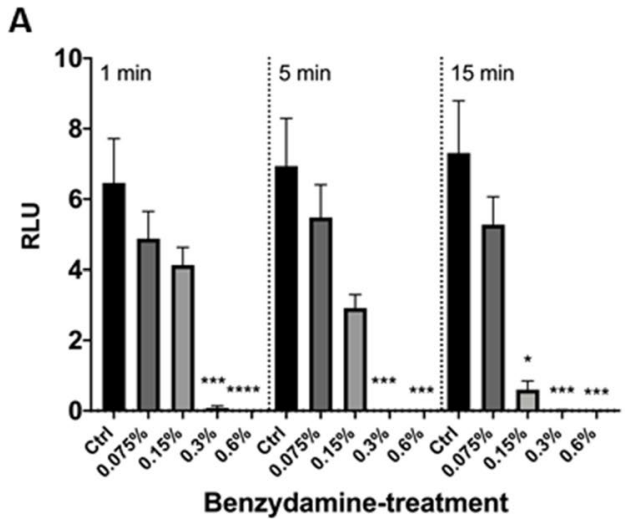

Fig. 3 Effects of benzydamine (A) and MoWs (B) treatments on the capacity of BLI-Candida to form a biofilm. Candida cells, whether pretreated with different concentrations of benzydamine or with different MoWs for 1,5 , or $15 \mathrm{~min}$, were tested for the ability to form a

\section{Effects of benzydamine and MoWs containing benzydamine on the persistence and regrowth of a 24-h-old C. albicans biofilm}

In order to test how benzydamine and MoWs would affect the capacity of a 24-h-old $C$. albicans biofilm to persist and possibly regrow, we used the experimental approach depicted in Fig. 1C. As shown in Fig. 4A, control Candida cells that had been treated with medium alone returned the highest bioluminescent signal at time $48 \mathrm{~h}$, indicating the strong capacity of the fungus to efficiently form a biofilm. When in parallel samples, the 24-h-old Candida biofilm had been treated with $0.15 \%, 0.3 \%$, and $0.6 \%$ benzydamine and further incubated for additional $24 \mathrm{~h}$ to allow residual biofilm regrowth, the bioluminescent signals were reduced in a highly significant fashion, irrespective of the contact times.

By employing the MoWs containing benzydamine 1 and 2 , the decreased bioluminescent signals revealed that the biofilms were indeed consistently affected, no matter whether 1, 5, or 15 min of treatment had been performed; yet statistically significant differences with the controls could be reached only when treating the biofilm with MoW 2 for $15 \mathrm{~min}$. As expected, the treatment of C. albicans 24-h-old biofilm with the placebo MoW 3 did not show any difference with the control group (Fig. 4B).

\section{Effects of benzydamine and MoWs containing benzydamine on a 48-h-old C. albicans biofilm}

Finally, the effects of benzydamine and MoWs containing benzydamine were assessed on a 48-h-old biofilm. The untreated Candida cells, embedded in a 48-h-old biofilm, returned the highest bioluminescent signal, once again confirming the strong capacity of the fungus to form efficiently

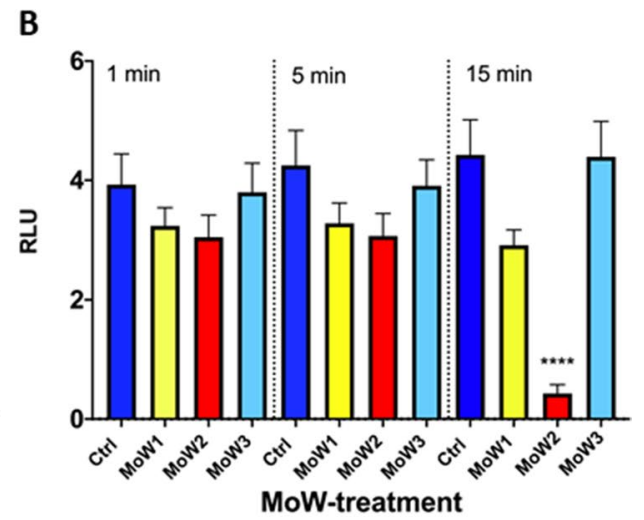

biofilm by bioluminescence assay. The results are shown as mean values \pm standard errors of triplicate samples from three different experiments. $* p \leq 0.05, * * * p<0.001$, or $* * * * p<0.0001$. RLU, relative luminescence units 


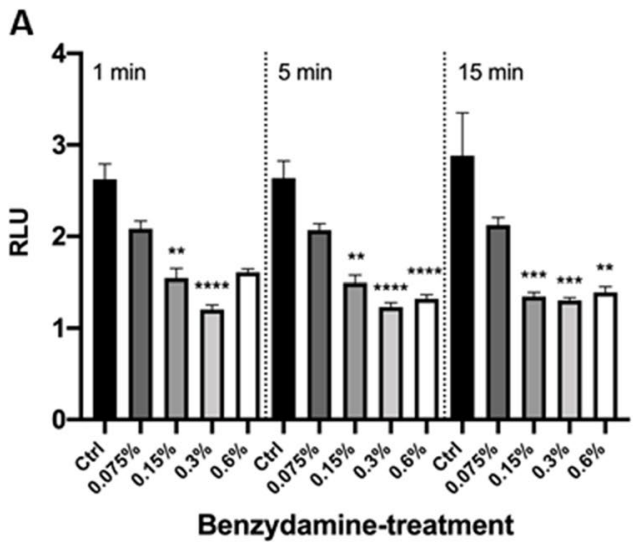

Fig. 4 Effects of benzydamine (A) and MoWs (B) treatments on a 24-h-old BLI-Candida biofilm persistence and regrowth. A 24-h-old Candida biofilm was exposed to benzydamine (A) and MoWs (B) treatments, for 1,5 , or $15 \mathrm{~min}$, washed, and further incubated for additional $24 \mathrm{~h}$ of incubation at $37^{\circ} \mathrm{C}$. Candida regrows in the fol-

a mature biofilm. The treatment of such 48-h-old Candida biofilm with $0.15 \%$ and $0.3 \%$ benzydamine returned a dosedependent decrease in bioluminescent signals, reaching statistical significance at $0.3 \%$ benzydamine treatment, whereas the treatment with $0.6 \%$ benzydamine produced a partial but non-significant reduction (Fig. 5A).

The treatment of the 48-h-old Candida biofilm with the MoWs reduced the bioluminescent signal to a different extent, depending on the MoW used (Fig. 5B). In particular, MoW 1 significantly impaired the preformed biofilm only when used for $15 \mathrm{~min}$; differently, MoW 2 treatment significantly reduced the bioluminescence signal, at all the times; finally, the biofilm treated with MoW 3 consistently returned bioluminescent signals similar to the control samples.

A

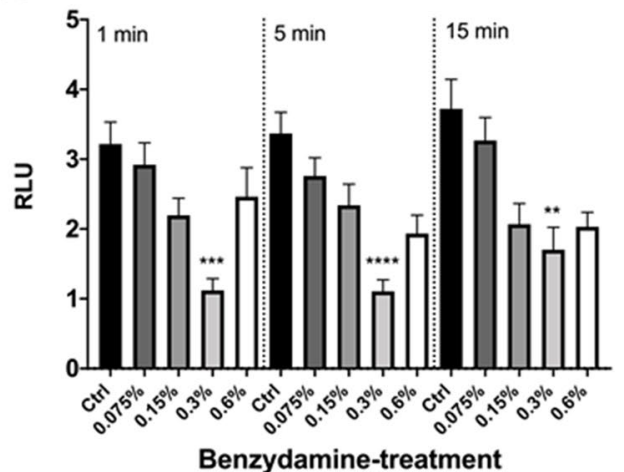

Fig. 5 Effects of benzydamine (A) or MoWs (B) treatments on a 48-h-old BLI-Candida biofilm. Benzydamine (A) or MoWs (B) were used against a 48-h-old Candida biofilm for 1, 5, or $15 \mathrm{~min}$; then, the amounts of residual biofilm were assessed by bioluminescence. The
B

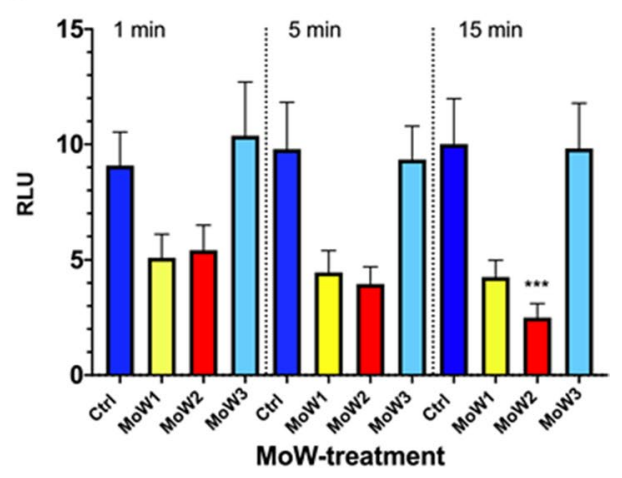

lowing $24 \mathrm{~h}$, as assessed by bioluminescence. The results are shown as mean values \pm standard errors of triplicate samples from three different experiments. $* * p<0.01, * * * p<0.001$, or $* * * * p<0.0001$. RLU, relative luminescence units

\section{Effects of benzydamine and MoWs containing benzydamine on fungal viability}

In order to verify if the contact with benzydamine or MoWs containing benzydamine could decrease fungal cell viability, the CFU counts were evaluated after contact between $\mathrm{Can}$ dida and benzydamine at various concentrations or MoWs. The results showed that, at the highest concentration tested $(0.6 \%)$, benzydamine significantly inhibited fungal viability, irrespective of the contact times, whereas the treatment with $0.3 \%$ benzydamine reduced fungal viability of $2 \log$ at all the contact times. No significant effects on fungal viability were observed after treatment with the lowest benzydamine concentrations, i.e., $0.15 \%$ and $0.075 \%$, irrespective of the contact times (Fig. 6A).

B

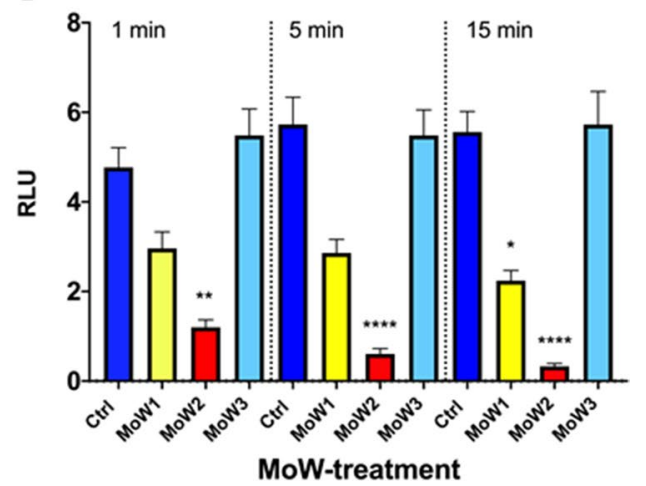

results are shown as mean values \pm standard errors of triplicate samples from three different experiments. ${ }^{*} p \leq 0.05$, ${ }^{*} p<0.01$, ${ }^{* * *} p<$ 0.001 , or $* * * * p<0.0001$. RLU, relative luminescence units 
The CFU counts after contact with the MoWs were also evaluated. The results showed that MoW 1 significantly inhibited fungal viability of about $1 \mathrm{Log}$, after 1 and $5 \mathrm{~min}$ of contact time, whereas a more pronounced effect on fungal viability could be observed after 15-min contact time (about $3 \mathrm{Log}$ ). The treatment with MoW 2 significantly reduced fungal viability of 1 and $3 \mathrm{Log}$, only after 5- and 15-min contact times, respectively. No significant effect on fungal viability was observed after treatment with the placebo MoW 3, being the CFU counts similar to those observed in the control group (Fig. 6B).

Then, the benzydamine MIC on BLI-C. albicans planktonic form was measured, according to the broth microdilution method NCCLS M27 [40]. We found that the MIC value $(0.0075 \mathrm{mg} / \mathrm{ml})$ corresponded to the lowest benzydamine dose employed in the above-described experiments $(0.075 \%)$. Such MIC value was confirmed also by the O.D. reading.

\section{Effects of MoWs containing benzydamine on quorum sensing (QS) molecules}

In order to unravel the mechanisms behind the inhibition of biofilm formation and biofilm regrowth by MoWs, we analyzed the levels of two Candida quorum sensing (QS) molecules, tyrosol and farnesol, by HPLC-ESI/HRMS.

Our results show that 15 -min treatment with MoWs 1 and 2 significantly impaired the production of tyrosol by Candida both during biofilm formation (Fig. 7A) and in the regrowth of a biofilm treated at time $24 \mathrm{~h}$ and further incubated in fresh medium up to $48 \mathrm{~h}$ (Fig. 7B). Differently, the treatment of Candida cells or Candida biofilm with the placebo MoW 3 did not impair tyrosol production, whose levels were comparable to those of the control samples.

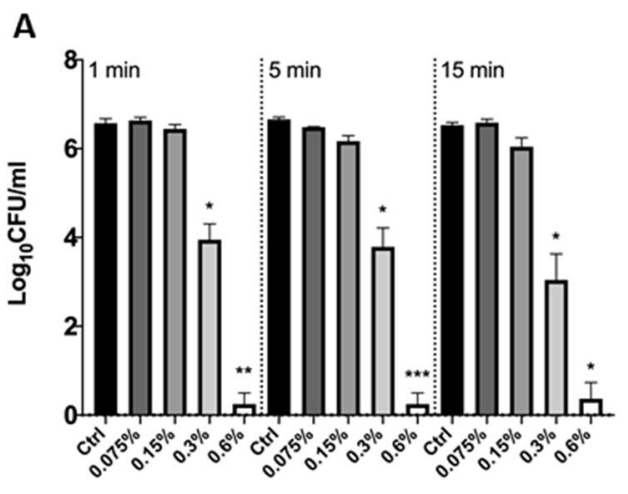

Benzydamine-treatment

Fig. 6 Effects of benzydamine (A) or MoWs (B) on C. albicans viability. Yeast cells were treated for 1,5 , or 15 min with benzydamine (A) from 0.075 to $0.6 \%$ ) and with MoWs (B); in all the cases, control cells were incubated in $\mathrm{cF} 12$ alone (controls). Then, the cells were washed, diluted with PBS, and seeded in SAB agar plates where they were let to grow at $37{ }^{\circ} \mathrm{C}$. After $48 \mathrm{~h}$, the CFU were counted,
In contrast, the analysis of farnesol levels consistently returned negative results (data not shown). This may be due to technical bias: either farnesol elution occurred very late, almost at the end of the process, and therefore it was not possible to reveal its signal, or the amounts of Candida-released farnesol were too low to be detected by the system.

\section{Discussion}

C. albicans is a fungal species, frequently colonizing the skin, the oral cavity, and the mucosal surfaces of several body sites in humans. Certain conditions, such as immunodeficiency $[1,2]$, diabetes [3], nutritional deficiencies [41], presence of dental prostheses [42], chemotherapeutic treatments [4, 5], and systemic steroid use [43], all contribute to turning this commensal fungus to an opportunistic pathogen [2, 44]. In particular, the capacity of $C$. albicans to adhere to biotic and abiotic surfaces represents the first and crucial step leading to biofilm formation $[22,45]$. By organizing itself as a sessile structure, $C$. albicans enhances its pathogenic potential, by increasing its resistance to immune-mediated defenses, to disinfectants, and antifungal drugs as well [10-14]. Interestingly, it has been recently demonstrated that the extracellular polysaccharides of $C$. albicans biofilm facilitate Streptococcus mutans colonization, persistence, and biofilm formation [46]. This ultimately suggests that the presence of the fungus in the oral cavity may contribute to enhance the risk of dental caries. Hence, the idea that by restraining Candida colonization through daily oral hygiene procedures (i.e., use of toothpaste, MoW, and dental floss) may help counteract also the colonization and persistence of specific oral pathogens, such as the cariogenic microorganism $S$. mutans.

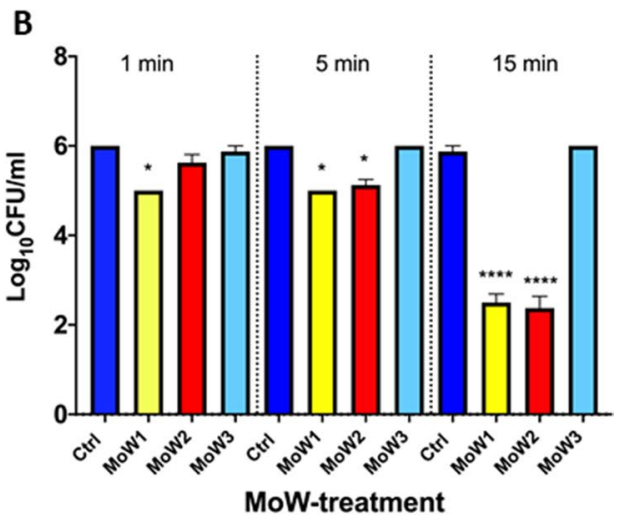

and the results were expressed as mean values of $\log (\mathrm{CFU} / \mathrm{ml}) \pm$ standard errors of duplicate samples from three different experiments. $* p \leq 0.05, * * p<0.01, * * * p<0.001$, or $* * * * p<0.0001$ indicate significant differences between benzydamine/MoWs-treated vs. $\mathrm{cF} 12$ treated C. albicans 


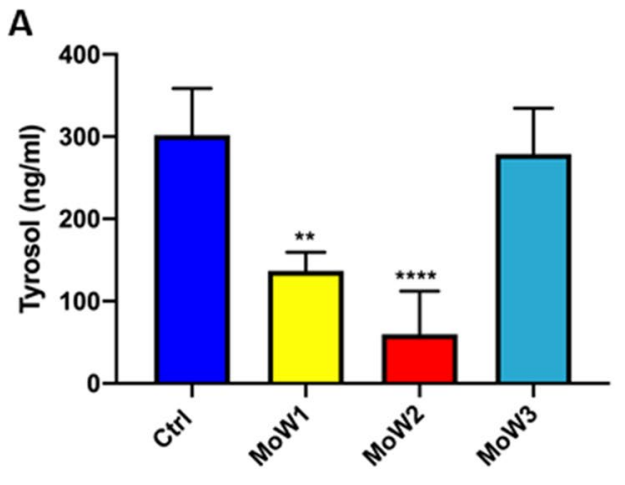

15' MoW-treatment

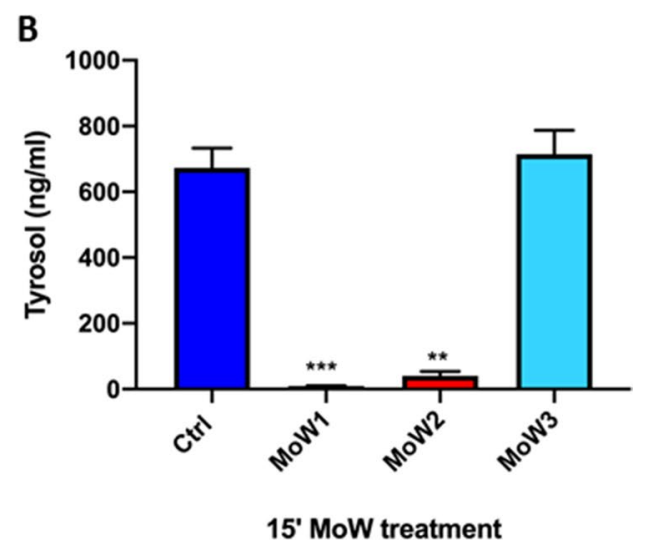

assessed on a 48-h-old Candida biofilm that had been treated at 24 $\mathrm{h}$ with the different MoWs for $15 \mathrm{~min}(\mathbf{B})$. The results are shown as mean values \pm standard errors of triplicate samples from three different experiments. $* * p<0.01, * * * p<0.001$, or $* * * * p<0.0001$

formation, and biofilm regrowth and persistence. Moreover, the effect on the preformed biofilm structure was also assessed. Since commercial MoWs contain either $0.15 \%$ or $0.3 \%$ benzydamine, such concentrations were chosen for the present study, in addition to lower $(0.075 \%)$ or higher $(0.6 \%)$ doses of the molecule. In addition, it is worth noting that the benzydamine MIC $(0.075 \%)$ does not have any effect on Candida yeast-to-hyphae transition and biofilm formation (data not shown). Accordingly, such concentration is the lowest employed in the present investigations.

The data presented here show that the treatment with benzydamine significantly impairs $C$. albicans capacity to adhere to plastic at all the contact times, when using $0.3 \%$ and $0.6 \%$ benzydamine. Differently, 5- and 15-min contact times have been necessary to impair $C$. albicans adhesion significantly when using $0.15 \%$ benzydamine. This result has been always consistent and, indeed, such impairment in adhesion capacity was observed when using benzydamine hydrochloride, both alone and included the formulations of MoWs 1 and 2. These in vitro data open to the possibility that, if also occurring in vivo, this benzydamine-mediated phenomenon may help prevent oral fungal colonization and persistence, especially in subjects, such as elderly denture wearers, quite frequently developing oral candidiasis [19, 20, 48].

The treatment of $C$. albicans with benzydamine inhibits significantly also biofilm formation at all the contact times, when using $0.3 \%$ and $0.6 \%$ benzydamine; differently, 15 -min contact time has been necessary to significantly inhibit biofilm formation when using $0.15 \%$ benzydamine. Such result, showing the impairment of biofilm formation when using the highest doses of benzydamine, is in line with the data obtained in the adhesion assay. This is not surprising, since the capacity of $C$. albicans to adhere to zydamine to interfere with $C$. albicans adhesion, biofilm 
surfaces (either biotic or abiotic) is the first stage of fungal colonization [22]; indeed, it easily allows local growth and leads to biofilm formation [45]. By assessing in parallel the effects of MoWs containing benzydamine on biofilm formation, both MoWs 1 and 2 have shown to be effective in reducing biofilm but only after 15 -min contact time, yet the statistical significance is only achieved with MoW 2. This latter result is not surprising since MoW 1 contains only benzydamine, while MoW 2 formulation includes cetylpyridinium chloride and benzydamine that, by acting in combination, likely achieve a better performance.

The 15-min contact time was chosen to verify the impact of benzydamine and MoWs on the production of QS molecules by Candida. The significant reduction in tyrosol, observed after treatment with both MoWs 1 and 2 , is in line with the results obtained by bioluminescence analysis and provides initial evidence that MoWs containing benzydamine are able to interfere in C. albicans QS system, at least in tyrosol production, in turn affecting the capacity of the fungus to form a biofilm.

To further investigate the impact of benzydamine on C. albicans biofilm, we used both the molecule alone and MoWs containing benzydamine against a preformed sessile fungal community ( $24 \mathrm{~h}$ old) and a mature ( $48 \mathrm{~h}$ old) biofilm. When analyzing the 24-h-old biofilm, a significant reduction has been found, by using benzydamine at any dose, or the MoWs 1 and 2, the latter providing the best effects. In parallel, the MS analysis of the corresponding supernatants reveals that the tyrosol production drastically drops upon treatment. This result implies that the MoWs damage C. albicans QS system in a very specific manner. Indeed, the lack of effects observed when treating with the placebo MoW 3 confirms that $C$. albicans $\mathrm{QS}$ system is affected by benzydamine and/or cetylpyridinium. To a similar extent, the 48-h-old $C$. albicans biofilm, upon treatment with $0.15 \%, 0.3 \%$, and $0.6 \%$ benzydamine, displays a significantly reduced viability. In addition, MoWs 1 and 2 show a significant effect in damaging a 48-h-old biofilm at all contact times, with the MoW 2 having the strongest effect. Again, the internal control, placebo MoW 3 , has no effects on the mature biofilm.

As assessed by CFU analysis, C. albicans cells are not killed by benzydamine, even though a significant decrement in viable cells can be observed upon treatment with $0.3 \%$ and, even more, with $0.6 \%$ benzydamine. To our opinion, these results argue that the decrement observed in adhesion and capacity to form biofilm stems from the impairment of such fungal virulence traits, rather than from $C$. albicans viability. From here, it may be speculated that benzydamine, as well as MoWs containing benzydamine 1 and 2, do not kill $C$. albicans, but they rather mitigate its virulence turning it back to a harmless commensal.
Overall, by means of a highly sensitive BLI-assay and HPLC-ESI/HRMS analysis of QS molecules, we have shown that $C$. albicans biofilm, in its different stages, is deeply impaired by benzydamine and MoWs containing benzydamine. Such effects happen to be achieved by affecting biofilm-embedded fungal cell virulence and metabolism, as demonstrated by the impaired production of the QS molecule tyrosol. These results are in line with the bioluminescence results, indicating that the inhibition of biofilm formation and biofilm regrowth could be explained by the reduced production of QS molecules, such as tyrosol, suggesting a selective role of MoWs in reducing Candida activity and metabolism. These findings may have relevant clinical implications, since the biofilm structure works as a shelter, providing the fungus with a protected environment, where resistance to immune-mediated defenses is enhanced [10] and susceptibility to antifungal drugs (such as fluconazole and amphotericin B) and disinfectants (like chlorhexidine) is greatly reduced [11-14].

In the light of this scenario, the present study documents the ability of benzydamine and MoWs containing benzydamine to counteract $C$. albicans biofilm formation as well as its regrowth and persistence. Such ability may provide an additional advantage for the overall oral health because it might interfere with the persistence of cariogenic pathogens within the oral cavity. Indeed, it has been reported that $C$. albicans and the cariogenic species $S$. mutans often coexist within the same dental biofilm, suggesting the establishment of a strict fungal-bacterial interaction, which in turn would facilitate the occurrence of dental caries [49-56]. Clinical studies further support the existence of a positive correlation between the prevalence of $C$. albicans in the oral cavity and the presence of $S$. mutans as well as the occurrence of dental caries in children $[51,57,58]$.

\section{Conclusion}

Benzydamine (both alone and included in MoWs) not only impairs fungal adhesion and biofilm formation, but it also affects biofilm persistence/regrowth and mature biofilm as well. These preliminary data have been obtained only from in vitro studies. If such results will be confirmed by future in vivo studies, MoWs containing benzydamine will provide an aid to prevent oral candidiasis, as well as a possible therapeutic tool to locally treat biofilm-related Candida infections. This possibility would become particularly important in several categories of patients, such as elderly denture wearers, immunocompromised individuals, and in general all those categories highly susceptible to develop oral Candida infections. Finally, given the positive interplay occurring between Candida and S. mutans in mixed biofilms, we may envisage that, by its ability to counteract 
C. albicans biofilm, benzydamine may also promote oral health indirectly by interfering with the colonization and persistence of cariogenic pathogens.

Supplementary Information The online version contains supplementary material available at https://doi.org/10.1007/s00784-021-04330-8.

Acknowledgements The authors acknowledge the "Fondazione Cassa di Risparmio di Modena" for funding the HPLC-ESI/HRMS QExactive system at the Centro Interdipartimentale Grandi Strumenti (CIGS) of the University of Modena and Reggio Emilia. We thank Cinzia Rosselli from the University of Modena and Reggio Emilia's "Centro Linguistico di Ateneo" for proof-reading the English text.

Funding This study was sponsored by Angelini Pharma S.p.A.

\section{Declarations}

\section{Ethics approval NA}

\section{Informed consent NA}

Conflict of interest Alessandra Capezzone de Joannon and Lorella Ragni are full-time employees of Angelini Pharma S.p.A.; at the time of research activities, Lucia Durando was also an employee of Angelini Pharma S.p.A.

Open Access This article is licensed under a Creative Commons Attribution 4.0 International License, which permits use, sharing, adaptation, distribution and reproduction in any medium or format, as long as you give appropriate credit to the original author(s) and the source, provide a link to the Creative Commons licence, and indicate if changes were made. The images or other third party material in this article are included in the article's Creative Commons licence, unless indicated otherwise in a credit line to the material. If material is not included in the article's Creative Commons licence and your intended use is not permitted by statutory regulation or exceeds the permitted use, you will need to obtain permission directly from the copyright holder. To view a copy of this licence, visit http://creativecommons.org/licenses/by/4.0/.

\section{References}

1. Thompson GR, Patel PK, Kirkpatrick WR et al (2010) Oropharyngeal candidiasis in the era of antiretroviral therapy. Oral Surg Oral Med Oral Pathol Oral Radiol Endod 109:488-495. https://doi.org/ 10.1016/j.tripleo.2009.11.026

2. Garcia-Cuesta C, Sarrion-Perez Mg, Bagan Jv (2014) Current treatment of oral candidiasis: a literature review. J Clin Exp Dent e576-e582 https://doi.org/10.4317/jced.51798

3. Mohammadi F, Javaheri MR, Nekoeian S, Dehghan P (2016) Identification of Candida species in the oral cavity of diabetic patients. Curr Med Mycol 2:1-7. https://doi.org/10.18869/acadpub.cmm.2. 2.4

4. Ramla S, Sharma V, Patel M (2016) Influence of cancer treatment on the Candida albicans isolated from the oral cavities of cancer patients. Support Care Cancer 24:2429-2436. https://doi.org/10. 1007/s00520-015-3035-8

5. Venkatasalu MR, Murang ZR, Ramasamy DTR, Dhaliwal JS (2020) Oral health problems among palliative and terminally ill patients: an integrated systematic review. BMC Oral Health 20:79. https://doi.org/10.1186/s12903-020-01075-w

6. Sudbery PE (2011) Growth of Candida albicans hyphae. Nat Rev Microbiol 9:737-748. https://doi.org/10.1038/nrmicro2636

7. Matsuura E, Godoy JSR, Bonfim-Mendonça $\mathrm{P}$ de $\mathrm{S}$ et al (2015) In vitro effect of Paullinia cupana (guaraná) on hydrophobicity, biofilm formation, and adhesion of Candida albicans' to polystyrene, composites, and buccal epithelial cells. Arch Oral Biol 60:471-478. https://doi.org/10.1016/j.archoralbio.2014.05.026

8. Moyes DL, Richardson JP, Naglik JR (2015) Candida albicansepithelial interactions and pathogenicity mechanisms: scratching the surface. Virulence 6:338-346. https://doi.org/10.1080/21505 594.2015.1012981

9. Ardizzoni A, Pericolini E, Paulone S et al (2018) In vitro effects of commercial mouthwashes on several virulence traits of Candida albicans, viridans streptococci and Enterococcus faecalis colonizing the oral cavity. PLoS One 13:e0207262. https://doi.org/10. 1371/journal.pone.0207262

10. Mayer FL, Wilson D, Hube B (2013) Candida albicans pathogenicity mechanisms. Virulence. Virulence 4:119-128. https:// doi.org/10.4161/viru. 22913

11. Tobudic S, Kratzer C, Lassnigg A, Presterl E (2012) Antifungal susceptibility of Candida albicans in biofilms: Candida albicans biofilm. Mycoses 55:199-204. https://doi.org/10.1111/j.14390507.2011.02076.x

12. Desai JV, Mitchell AP (2015) Candida albicans biofilm development and its genetic control. Microbiol Spectr 3. https://doi.org/ 10.1128/microbiolspec.MB-0005-2014

13. Rahmani-Badi A, Sepehr S, Babaie-Naiej H (2015) A combination of cis-2-decenoic acid and chlorhexidine removes dental plaque. Arch Oral Biol 60:1655-1661. https://doi.org/10.1016/j.archo ralbio.2015.08.006

14. Uygun-Can B, Kadir T, Gumru B (2016) Effect of oral antiseptic agents on phospholipase and proteinase enzymes of Candida albicans. Arch Oral Biol 62:20-27. https://doi.org/10.1016/j.archo ralbio.2015.11.006

15. Kovács R, Majoros L (2020) Fungal quorum-sensing molecules: a review of their antifungal effect against Candida biofilms. J Fungi (Basel) 6. https://doi.org/10.3390/jof6030099

16. Hornby JM, Jensen EC, Lisec AD et al (2001) Quorum sensing in the dimorphic fungus Candida albicans is mediated by farnesol. Appl Environ Microbiol 67:2982-2992. https://doi.org/10.1128/ AEM.67.7.2982-2992.2001

17. Wongsuk T, Pumeesat P, Luplertlop N (2016) Fungal quorum sensing molecules: role in fungal morphogenesis and pathogenicity. J Basic Microbiol 56:440-447. https://doi.org/10.1002/jobm. 201500759

18. Albuquerque $\mathrm{P}$, Casadevall A (2012) Quorum sensing in fungi-a review. Med Mycol 50:337-345. https://doi.org/10.3109/13693 786.2011.652201

19. He XY, Meurman JH, Kari K et al (2006) In vitro adhesion of Candida species to denture base materials. Mycoses 49:80-84. https://doi.org/10.1111/j.1439-0507.2006.01189.x

20. de Souza RD, Mores AU, Cavalca L et al (2009) Cell surface hydrophobicity of Candida albicans isolated from elder patients undergoing denture-related candidosis. Gerodontology 26:157161. https://doi.org/10.1111/j.1741-2358.2008.00229.x

21. Hagg U (2004) The effect of fixed orthodontic appliances on the oral carriage of Candida species and Enterobacteriaceae. Eur J Orthod 26:623-629. https://doi.org/10.1093/ejo/26.6.623

22. Bürgers R, Schneider-Brachert W, Rosentritt M et al (2009) Candida albicans adhesion to composite resin materials. Clin Oral Investig 13:293-299. https://doi.org/10.1007/s00784-008-0226-4

23. Ruhnke M (2002) Skin and mucous membrane infections. In: Calderone RA (ed) Candida and candidiasis. ASM Press, Washington D.C., pp 307-325 
24. Haerian-Ardakani A, Rezaei M, Talebi-Ardakani M et al (2015) Comparison of antimicrobial effects of three different mouthwashes. Iran J Public Health 44:997-1003

25. Paulone S, Malavasi G, Ardizzoni A et al (2017) Candida albicans survival, growth and biofilm formation are differently affected by mouthwashes: an in vitro study. New Microbiol 40:45-52

26. Sironi M, Pozzi P, Polentarutti N et al (1996) Inhibition of inflammatory cytokine production and protection against endotoxin toxicity by benzydamine. Cytokine 8:710-716. https://doi.org/ 10.1006/cyto. 1996.0094

27. Sironi M, Milanese C, Vecchi A et al (1997) Benzydamine inhibits the release of tumor necrosis factor- $\alpha$ and monocyte chemotactic protein- 1 by Candida albicans-stimulated human peripheral blood cells. Int J Clin Lab Res 27:118-122. https://doi.org/10. 1007/BF02912445

28. Cheng KKF (2004) Children's acceptance and tolerance of chlorhexidine and benzydamine oral rinses in the treatment of chemotherapy-induced oropharyngeal mucositis. Eur J Oncol Nurs 8:341-349. https://doi.org/10.1016/j.ejon.2004.04.002

29 Epstein JB, Stevenson-Moore P, Jackson S et al (1989) Prevention of oral mucositis in radiation therapy: a controlled study with benzydamine hydrochloride rinse. Int J Radiat Oncol Biol Phys 16:1571-1575. https://doi.org/10.1016/0360-3016(89)90964-4

30. Kim JH, Chu FC, Lakshmi V, Houde R (1986) Benzydamine HCl, a new agent for the treatment of radiation mucositis of the oropharynx. Am J Clin Oncol 9:132-134. https://doi.org/10.1097/ 00000421-198604000-00006

31. Pina-Vaz C, Rodrigues AG, Sansonetty F et al (2000) Antifungal activity of local anesthetics against Candida species. Infect Dis Obstet Gynecol 8:124-137. https://doi.org/10.1155/S106474490 0000168

32. Roopashri G, Jayanthi K, Guruprasad R (2011) Efficacy of benzydamine hydrochloride, chlorhexidine, and povidone iodine in the treatment of oral mucositis among patients undergoing radiotherapy in head and neck malignancies: a drug trail. Contemp Clin Dent 2:8. https://doi.org/10.4103/0976-237X.79292

33. Kazemian A, Kamian S, Aghili M et al (2009) Benzydamine for prophylaxis of radiation-induced oral mucositis in head and neck cancers: a double-blind placebo-controlled randomized clinical trial. Eur J Cancer Care (Engl) 18:174-178. https://doi.org/10. 1111/j.1365-2354.2008.00943.x

34. Epstein JB, Silverman S, Paggiarino DA et al (2001) Benzydamine $\mathrm{HCl}$ for prophylaxis of radiation-induced oral mucositis: results from a multicenter, randomized, double-blind, placebo-controlled clinical trial. Cancer 92:875-885. https://doi.org/10.1002/10970142(20010815)92:4\%3c875::aid-cncr1396\%3e3.0.co;2-1

35. Bozkurt FY, Öztürk M, Yetkin Z (2005) The effects of three oral sprays on plaque and gingival inflammation. J Periodontol 76:1654-1660. https://doi.org/10.1902/jop.2005.76.10.1654

36. Seshan H, Shanavas S, Ashwini S (2016) Effective evaluation of benzydamine hydrochloride as a mouth wash in subjects with plaque induced gingival inflammation. Int J Oral Health Dent 2:161-170

37. Haydari M, Bardakci AG, Koldsland OC et al (2017) Comparing the effect of $0.06 \%-, 0.12 \%$ and $0.2 \%$ chlorhexidine on plaque, bleeding and side effects in an experimental gingivitis model: a parallel group, double masked randomized clinical trial. BMC Oral Health 17:118. https://doi.org/10.1186/s12903-017-0400-7

38. Herrera D, Santos S, Ferrus J et al (2005) Efficacy of a $0.15 \%$ benzydamine hydrochloride and $0.05 \%$ cetylpyridinium chloride mouth rinse on 4-day de novo plaque formation. J Clin Periodontol 32:595-603. https://doi.org/10.1111/j.1600-051X.2005. 00718.x

39. Enjalbert B, Rachini A, Vediyappan G et al (2009) A multifunctional, synthetic Gaussia princeps luciferase reporter for live imaging of Candida albicans infections. Infect Immun 77:48474858. https://doi.org/10.1128/IAI.00223-09

40. CLSI (2017) Reference method for broth dilution antifungal susceptibility testing of yeasts, 4th edn. CLSI Standard M27. Clinical and Laboratory Standard Institute, Wayne

41. Paillaud E, Merlier I, Dupeyron C et al (2004) Oral candidiasis and nutritional deficiencies in elderly hospitalised patients. Br J Nutr 92:861-867. https://doi.org/10.1079/bjn20041264

42. Bianchi CMP de C, Bianchi HA, Tadano T et al (2016) Factors related to oral candidiasis in elderly users and non-users of removable dental prostheses. Rev Inst Med Trop Sao Paulo 58:17. https://doi.org/10.1590/S1678-9946201658017

43. Buhl R (2006) Local oropharyngeal side effects of inhaled corticosteroids in patients with asthma. Allergy 61:518-526. https:// doi.org/10.1111/j.1398-9995.2006.01090.x

44. Lalla RV, Patton LL, Dongari-Bagtzoglou A (2013) Oral candidiasis: pathogenesis, clinical presentation, diagnosis and treatment strategies. J Calif Dent Assoc 41:263-268

45. Douglas LJ (2003) Candida biofilms and their role in infection. Trends Microbiol 11:30-36. https://doi.org/10.1016/s0966$842 x(02) 00002-1$

46. Khoury ZH, Vila T, Puthran TR et al (2020) The role of Candida albicans secreted polysaccharides in augmenting Streptococcus mutans adherence and mixed biofilm formation: in vitro and in vivo studies. Front Microbiol 11:307. https://doi.org/10.3389/ fmicb.2020.00307

47. Nicolatou-Galitis O, Sarri T, Bowen J et al (2013) Systematic review of anti-inflammatory agents for the management of oral mucositis in cancer patients. Support Care Cancer 21:3179-3189. https://doi.org/10.1007/s00520-013-1847-y

48. Figueiral MH, Azul A, Pinto E et al (2007) Denture-related stomatitis: identification of aetiological and predisposing factors ? a large cohort. J Oral Rehabil 34:448-455. https://doi.org/10.1111/j. 1365-2842.2007.01709.x

49 Barbieri D de SV, Vicente VA, Fraiz FC et al (2007) Analysis of the in vitro adherence of Streptococcus mutans and Candida albicans. Braz J Microbiol 38:624-631. https://doi.org/10.1590/ S1517-83822007000400009

50. Jarosz LM, Deng DM, van der Mei HC et al (2009) Streptococcus mutans competence-stimulating peptide inhibits Candida albicans hypha formation. Eukaryot Cell 8:1658-1664. https://doi.org/10. 1128/EC.00070-09

51. Raja M, Hannan A, Ali K (2010) Association of oral candidal carriage with dental caries in children. Caries Res 44:272-276. https://doi.org/10.1159/000314675

52. Sztajer H, Szafranski SP, Tomasch J et al (2014) Cross-feeding and interkingdom communication in dual-species biofilms of Streptococcus mutans and Candida albicans. ISME J 8:22562271. https://doi.org/10.1038/ismej.2014.73

53. Hwang G, Marsh G, Gao L et al (2015) Binding force dynamics of Streptococcus mutans-glucosyltransferase B to Candida albicans. J Dent Res 94:1310-1317. https://doi.org/10.1177/0022034515 592859

54. Hwang G, Liu Y, Kim D et al (2017) Candida albicans mannans mediate Streptococcus mutans exoenzyme GtfB binding to modulate cross-kingdom biofilm development in vivo. PLoS Pathog 13:e1006407. https://doi.org/10.1371/journal.ppat.1006407

55. Yang C, Scoffield J, Wu R et al (2018) Antigen I/II mediates interactions between Streptococcus mutans and Candida albicans. Mol Oral Microbiol 33:283-291. https://doi.org/10.1111/omi.12223

56. Lobo CIV, Rinaldi TB, Christiano CMS et al (2019) Dual-species biofilms of Streptococcus mutans and Candida albicans exhibit more biomass and are mutually beneficial compared with singlespecies biofilms. J Oral Microbiol 11:1581520. https://doi.org/10. 1080/20002297.2019.1581520 
57. de Carvalho FG, Silva DS, Hebling J et al (2006) Presence of mutans streptococci and Candida spp. in dental plaque/dentine of carious teeth and early childhood caries. Arch Oral Biol 51:10241028. https://doi.org/10.1016/j.archoralbio.2006.06.001

58. Fragkou S, Balasouli C, Tsuzukibashi O et al (2016) Streptococcus mutans, Streptococcus sobrinus and Candida albicans in oral samples from caries-free and caries-active children. Eur Arch Paediatr Dent 17:367-375. https://doi.org/10.1007/s40368-016-0239-7
Publisher's note Springer Nature remains neutral with regard to jurisdictional claims in published maps and institutional affiliations. 